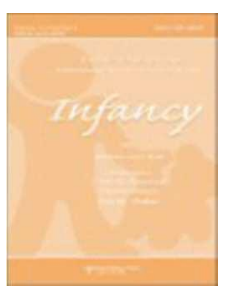

\title{
The emergence and stability of the attentional bias to fearful faces in infancy
}

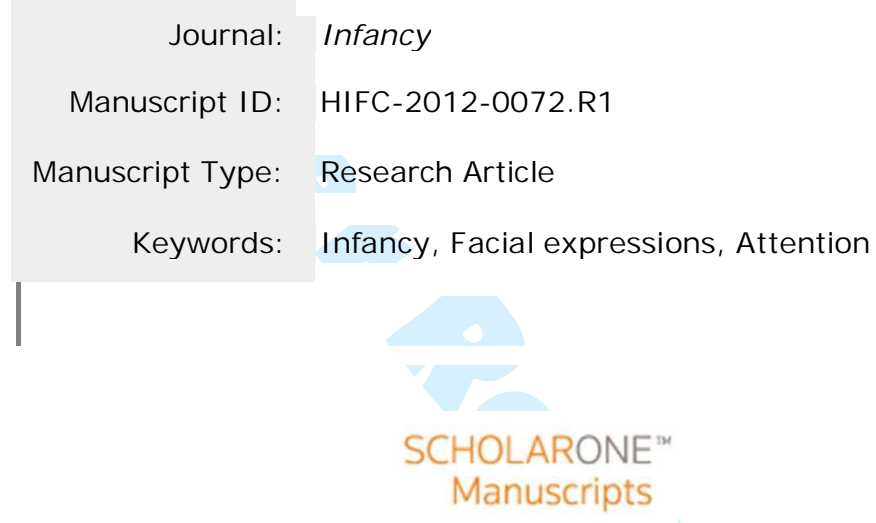

Authors: Mikko J. Peltola, Jari K. Hietanen, Linda Forssman, Jukka M Leppänen 


\begin{abstract}
Several studies have shown that at 7 months of age, infants display an attentional bias toward fearful facial expressions. In the present study, we analyzed visual attention and heart rate data from a cross-sectional study with 5-, 7-, 9-, and 11-month-old infants (Experiment 1) and visual attention from a longitudinal study with 5- and 7-month-old infants (Experiment 2) to examine the emergence and stability of the attentional bias to fearful facial expressions. In both experiments, the attentional bias to fearful faces appeared to emerge between 5 and 7 months of age: 5-month-olds did not show a difference in disengaging attention from fearful and non-fearful faces, whereas 7- and 9month-old infants had a lower probability of disengaging attention from fearful than nonfearful faces. Across the age groups, heart rate (HR) data (Experiment 1) showed a more pronounced and longer-lasting HR deceleration to fearful than non-fearful expressions.

The results are discussed in relation to the development of the perception and experience of fear and the interaction between emotional and attentional processes.
\end{abstract}




\section{The emergence and stability of the attentional bias to fearful faces in infancy}

One of the developmental challenges infants face during the first year of life is to acquire the ability to use emotional information from others' nonverbal signals for the service of behavioral regulation and learning about novel and potentially threatening situations. Potential early precursors to the ability to learn from others' emotional signals may be observed in young infants' selective attentional responses to facial expressions. A number of studies have shown that during the second half of the first year of life, infants preferentially attend to fearful facial expressions over positive and neutral emotion cues (Leppänen \& Nelson, 2009). It is likely that an attentional bias toward fearful expressions over other stimuli fosters associative learning in situations involving expressions of fear and impending danger. When two different facial expressions are presented side by side on a computer screen for a period of 10-20 seconds in a visual paired comparison (VPC)

task, 7-month-old infants spontaneously look longer at fearful than at happy faces (Kotsoni, de Haan, \& Johnson, 2001; Nelson \& Dolgin, 1985). Using electroencephalography (EEG), Nelson and de Haan (1996) observed that in 7-month-old infants, an event-related brain potential (ERP) component associated with allocating attention to visual stimuli (i.e., the Nc component) was larger for fearful than happy faces, indicating increased attention to fearful faces (for similar findings, see also de Haan, Belsky, Reid, Volein, \& Johnson, 2004; Grossmann et al., 2011; Leppänen, Moulson, Vogel-Farley, \& Nelson, 2007).

Previous studies have used a modification of the Overlap paradigm (Aslin \& Salapatek, 1975) to further understand infants' attentional biases and physiological responses toward emotional expressions. In the Overlap paradigm, non-emotional stimuli 
are presented in the visual periphery to attract attention to their location after attention has first been engaged on a centrally presented facial expression stimulus. The pattern of results emerging from these studies is that infants aged 7 months and older are less likely (Nakagawa \& Sukigara, 2012; Peltola, Leppänen, Palokangas, \& Hietanen, 2008) and slower (Peltola, Leppänen, Vogel-Farley, Hietanen, \& Nelson, 2009) to disengage their attention away from fearful than from happy and neutral faces or control stimuli matched for low-level visual properties. Studies measuring physiological responses with heart rate (HR) recording have further shown that the attentional bias to fearful faces is associated with an increased deceleration of HR (Leppänen et al., 2010; Peltola, Leppänen, \& Hietanen, 2011) which is suggested to reflect a physiological correlate of enhanced attentional and sensory processing (Bradley, 2009; Reynolds \& Richards, 2007). The reasons for the increased attentiveness to facial signals of fear at the expense of other stimuli are not perfectly clear. Some authors (e.g., Kagan \& Herschkowitz, 2005; Nelson \& de Haan, 1996; Vaish, Grossmann, \& Woodward, 2008) have raised the possibility that instead of a response to the emotional content of the fearful expression, the attentional bias to fearful faces that has been observed with diverse methods (i.e., looking times, ERPs, and HR) may reflect a novelty preference (i.e., the fact that facial expressions of fear are relatively rare in young infants' rearing environment; Malatesta \& Haviland, 1982) or attention capture by salient low-level visual features such as the increased exposure of the sclera (eye white) in fearful expressions. Studies using the Overlap paradigm have shown, however, that the attentional bias is not simply reducible to these types of factors. For example, facial stimuli that contain novel (i.e., non-emotional grimaces) and distinctive visual features (i.e., fearful eyes embedded within an otherwise 
neutral expression) do not attract attention in a similar fashion as fearful faces do (Peltola

et al., 2008; Peltola, Leppänen, Vogel-Farley et al., 2009).

Although studies employing ERP, HR, and eye movement measures have repeatedly shown an attentional bias to fearful faces in 7-month-old infants, the developmental time-course of the attentional bias has been considerably less well documented. Available evidence indicates, however, that the enhanced attentiveness toward fearful expressions emerges between 5 and 7 months of age. Peltola, Leppänen, Mäki, and Hietanen (2009) analyzed attention-related ERPs and looking times from 5and 7-month-old infants while they were shown fearful and happy faces. For the 7month-old infants, the results replicated earlier studies in that the ERP responses were larger and the looking times longer for fearful than happy faces. No significant differences were observed with either measure in 5-month-old infants. By measuring attention-related ERPs to neutral and fearful faces with the eyes looking toward or away from a laterally presented object, Hoehl and Striano (2010) found no differences in the magnitude of the ERP responses between the two expressions in 3-month-old infants, whereas in 6-month-old infants the responses were larger to fearful faces with the eyes looking toward the object. Bornstein and Arterberry (2003) also did not observe differences in 5-month-olds' looking times to fearful and happy faces. Vaish et al. (2008) even argued for the existence of a "positivity bias" during the first six months as there are findings of longer looking times to happy than fearful expressions in newborns (Farroni, Menon, Rigato, \& Johnson, 2007) and happy compared to angry expressions in 4- to 6month-old infants (LaBarbera, Izard, Vietze, \& Parisi, 1976; Wilcox \& Clayton, 1968). Together, these findings suggest a change in neural responsiveness and relative weighting 
of attention to fear-related signals at around 6 months of age. Whether or not the enhanced responsiveness to fear-related signals is an enduring effect that displaces the early positivity bias is unclear. Todd, Evans, Morris, Lewis, and Taylor (2011), for example, showed that in 4- to 6-year-old children amygdala activation was stronger to happy than angry faces, indicating preferential responding to positive emotional signals over other emotionally salient signals in early childhood.

Besides eye movement based measures of attentional bias for fear, it is important to measure possible age-related changes in physiological measures indexing enhanced attentional and sensory processing. Therefore, recording of electrocardiography (ECG) was included in Experiment 1. Orienting of attention to external stimuli is typically accompanied by a rapid HR deceleration in adults (Graham \& Clifton, 1966) and in infants (Reynolds \& Richards, 2007). The magnitude and the duration of HR deceleration during presentation of stimuli in the Overlap paradigm have also been used as measures of attentiveness and depth of encoding of the central stimulus (Blaga \& Colombo, 2006; Reynolds \& Richards, 2007). In adults, the HR deceleration response is augmented by emotionally negative stimuli, such as unpleasant scenes or angry faces (Bradley, Lang, \& Cuthbert, 1993; Bradley, 2009; Kolassa \& Miltner, 2006; Lang \& Bradley, 2010). Bradley (2009) regarded the momentary threat-related slowing of HR as a part of an automatic defense-related orienting reflex that acts to facilitate perceptual processing and extraction of information about potentially significant stimuli. While research on similar adult-like emotional enhancement of the HR response in young children is scarce, two studies measuring HR from 7-month-old infants during the Overlap paradigm showed a correspondence between HR and behavioral measures of attentional bias to fearful faces 
in that the HR orienting response (i.e., deceleration) was larger during trials with fearful than happy/neutral faces (Leppänen et al., 2010; Peltola et al., 2011). These findings led us to test the hypothesis that the threat-related modulation of HR deceleration follows a similar developmental course as the attention measures based on eye movements.

The aim of the present study was to further delineate the developmental course of attention to fearful faces. To this end, we pooled data from cross-sectional studies conducted with 5-, 7-, 9-, and 11-month-old infants (Experiment 1) and an ongoing longitudinal study with 5- and 7-month-old infants (Experiment 2) to examine age-related changes in attention to fearful expressions. The experimental setups varied slightly between Experiment 1 and 2, but in both experiments, data were available for infants' attention to neutral, happy, and fearful facial expressions measured with the Overlap paradigm. The ability to disengage and shift attention from an attended to another stimulus is sufficiently developed by the age of 5 months (e.g., Colombo, 2001; Hunnius \& Geuze, 2004) to consider the Overlap paradigm suitable for the age range in question. Blaga and Colombo (2006), for example, provided evidence that performance in the Overlap paradigm with non-emotional stimuli does not markedly change from 4 to 7 months of age. The first aim was to investigate whether the change in attentiveness to fearful faces between 5 and 7 months of age would be replicated using the Overlap paradigm. In the Overlap paradigm, the attentional bias should manifest in a lower probability of disengaging attention from fearful than happy and neutral faces as well as in larger HR deceleration during fearful face trials. Second, it was of interest to test whether the attentional bias to fearful faces remains present until the end of the first year. Although studies using comparable methods with infants older than 7 months of age are 
limited, the available evidence points to a continuing attentional preference toward fearful faces. Using a visual paired comparison task, LoBue and DeLoache (2010) demonstrated longer looking times for fearful than happy faces in a group consisting of 8to 14-month-old infants. Hoehl and Striano (2010) observed enhanced attention-related ERP responses for fearful relative to neutral faces in 9-month-old infants. Recently, Nakagawa and Sukigara (2012), using a slightly modified version of the Overlap paradigm, found that infants show a lower probability of disengaging attention from fearful than happy and neutral faces from 12 to 36 months of age. These data led us to expect similar modulation of attention by fearful faces in the Overlap paradigm in infants aged 7,9 , and 11 months.

To summarize, we analyzed both cross-sectional and longitudinal datasets in the present study to chart the emergence and stability of the attentional bias toward fearful faces in infancy. We hypothesized that the attentional bias to fearful faces would emerge between 5 and 7 months of age and remain present until 11 months of age. The attentional bias should manifest in a lower probability of disengaging attention away from fearful than happy and neutral faces (Experiments 1 and 2) as well as in larger HR deceleration during fearful face trials (Experiment 1).

\section{Experiment 1}

\section{Methods}

Participants. Experiment 1 is based on data from a previous study with 7-monthold infants (reported in Peltola et al., 2011) and new data collected from 5-, 9-, and 11month-old infants. The full sample consisted of 113 infants in separate age groups of 5month-olds $(n=29$, mean age $=153$ days, $S D=4.14,16$ girls $), 7$-month-olds $(n=29$, 
mean age $=216$ days, $S D=4.41,8$ girls $), 9$-month-olds $(n=29$, mean age $=274$ days, $S D=3.79,10$ girls $)$, and 11-month-olds $(n=26$, mean age $=336$ days, $S D=2.93,12$ girls). All of the participants came from urban, middle class families of Finnish origin. Apart from two infants who were excluded from the analyses due to pre-term birth (one 5-month-old and one 7-month-old), the infants were born full term ( $\geq 37$ weeks), had a birth weight of $>2400 \mathrm{~g}$ and no history of visual or neurological abnormalities. After excluding some infants from the final data analyses ${ }^{1}, 104$ infants remained in the disengagement probability analyses (5-month-olds: $n=25$; 7-month-olds: $n=26$; 9month-olds: $n=28$; 11-month-olds: $n=25$ ) and 91 infants remained in the HR analyses (5-month-olds: $n=24 ; 7$-month-olds: $n=19 ; 9$-month-olds: $n=27 ; 11$-month-olds: $n=$ 21). Approval for the project was obtained from the University of Tampere and Tampere Area Ethical Review Board and an informed, written consent was obtained from the parent of each child.

Stimuli and procedure. The stimuli were color images of neutral, happy, and fearful facial expressions of two female models. With an approximately $60-\mathrm{cm}$ viewing distance, the faces measured $15.4^{\circ}$ and $10.8^{\circ}$ of vertical and horizontal visual angle, respectively. Prior to data collection, a group of adults $(n=18)$ rated the facial expressions for happiness and fearfulness on a scale from 1 to 7 . The ratings confirmed that the happy $(M=5.9)$ and fearful $(M=6.2)$ facial expressions used were considered good examples of the respective emotions. In addition, 3 to 4 trials with a monkey face were also presented during the later parts of the testing session as occasional attentiongetters. The data for the monkey stimuli were not analyzed. 
Infants were seated on the parent's lap in a dimly lit room while stimuli were presented on a 19-inch computer monitor that was surrounded by black panels. A video camera hidden above the monitor recorded the infant's face and enabled the experimenter to control stimulus presentation. Infants' heart rate and eye movements were measured during the Overlap paradigm (Figure 1). A fixation image (an animated underwater scene from the movie "Finding Nemo") preceded each trial. Once the infant's attention was focused on the fixation image, one of the face stimuli was presented on the center of the screen on a white background. After $700 \mathrm{~ms}$ from the onset of the face stimulus, the face was flanked by a peripheral stimulus presented $13.6^{\circ}$ equiprobably on the left or right for $2000 \mathrm{~ms}$. The peripheral stimuli were black-and-white vertically arranged circles or a checkerboard pattern, measuring $15.4^{\circ}$ and $4.3^{\circ}$ vertically and horizontally, respectively. Every infant saw only one model's face.

During the first 15 trials, neutral, happy, and fearful expressions were presented in random order with the constraint that the same expression was presented no more than twice in a row and the flanker on the same side of the screen no more than three times in a row. After the first 15 trials, only happy and fearful expressions were presented until the infant became inattentive or fussy. The latter trials were added for the specific purposes of simultaneous ECG, EEG, and eye movement recording. The EEG was eventually recorded only from the 7-month-old infants and is not reported here. Therefore, the present analyses are based on the first 15 trials of the experiment.

\author{
Insert Figure 1 about here
}


Analysis of the behavioral data. An independent observer coded the videos with frame-by-frame playback using VirtualDubMod 1.5.10.2. Trials including excessive movement, anticipatory eye movements (eye movements commenced within $160 \mathrm{~ms}$ after the onset of the peripheral stimulus), and eye movements away from the face that were not directed towards the peripheral stimulus were excluded from the analyses. The mean number of scorable trials included in the analyses was 13.16 (neutral $=4.40$; happy $=$ 4.37; fearful $=4.38$ ), with no significant differences in the number of scorable trials between the age groups, $F(3,101)=1.85, p>.14$. The mean numbers of trials are comparable to earlier studies using the Overlap paradigm (Leppänen et al., 2011; Peltola et al., 2008; Peltola, Leppänen, Vogel-Farley et al., 2009).

For the behavioral data, disengagement probability from fearful, happy, and neutral faces was analyzed. It was calculated as the proportion of trials with a saccade toward the peripheral stimulus out of the total number of scorable trials (i.e., a sum of trials with a saccade toward the peripheral stimulus as well as no-saccade trials on which the child did not shift attention away from the face during the whole trial). Analyses of attention disengagement latencies are not reported because a large number of infants failed to provide a minimum of 2 trials with saccades away from the central stimulus in each stimulus condition. To establish the reliability of the data coding procedures, another independent observer who was blind to the stimulus condition coded the data from 28 subjects ( $25 \%$; 7 infants within each age group). The interobserver agreement (Cohen's Kappa) for the classification of responses to different response categories (i.e., correct saccade present, saccade absent, non-scorable trial) was on average .94. 


\section{Acquisition and analysis of the HR data. Electrocardiogram (ECG) was}

recorded throughout the task with two pre-gelled and self-adhesive electrodes placed on the participant's chest. The ECG was band-pass filtered from 0.05 to $30 \mathrm{~Hz}$, amplified with a gain of 1000 (range $+/-2750 \mu \mathrm{V}$; Accuracy .084 $\mu \mathrm{V} / \mathrm{LSB})$, and stored on a computer disk at the sample rate of $1000 \mathrm{~Hz}$ (Neuroscan/Synamps). Offline, the data were analyzed by using an in-house (Matlab-based) algorithm to identify QRS complexes in the ECG signal, and to measure the time intervals between two successive R-waves (i.e., interbeat intervals or IBI). Lengthening of the IBI corresponds to HR deceleration and shortening to HR acceleration. After an algorithm-based detection of R-peaks, the data were manually corrected for falsely detected and missing peaks. The same trials that were removed from the eye movement analyses due to anticipatory eye movements and eye movements away from the face that were not directed towards the peripheral stimulus were also excluded from the HR analyses. Additional trials were removed due to excessive movement causing distortion in the ECG signal. To be included in the statistical analyses of the HR data, a minimum of 2 good trials were required for each stimulus condition. The mean number of trials retained for the HR analyses was 13.08 $($ neutral $=4.37$; happy $=4.34$; fearful $=4.36)$, with no significant differences between the age groups, $F(3,90)=2.01, p>.12$. For a period between $500 \mathrm{~ms}$ pre-stimulus and 2500 ms post-stimulus within each trial, the IBIs were quantified and assigned to 500-ms intervals by weighting each IBI by the proportion of the 500-ms interval occupied by that IBI (see Richards \& Turner, 2001). Finally, IBIs were converted to beats per minute (BPM) and averaged across different trials within each stimulus condition. The analyses were performed with HR change scores which were calculated by subtracting the BPMs 
of each post-stimulus 500-ms interval from the BPM during the pre-stimulus period.

Accordingly, negative change score values indicate HR deceleration while positive values indicate HR acceleration during the trial.

Statistical analyses. As the eye movement data were not normally distributed, the analyses of the behavioral data were conducted using nonparametric methods. First, to provide data on potential age differences in the overall attention disengagement from facial stimuli, age differences in disengagement probability across facial expressions were analyzed with the Kruskal-Wallis test and Mann-Whitney U-tests were used for pairwise comparisons between age groups. Second, the effects of facial expressions on disengagement probability were analyzed within each age group separately by using Friedman tests for main effect analyses and Wilcoxon tests for pairwise comparisons. Third, a fear bias score was calculated to further examine the potential age differences in the magnitude of relative attentional bias to fearful faces. The fear bias score was calculated by subtracting the disengagement probability score for fearful faces from the average disengagement probability score for happy and neutral faces. Thus, the bias score reflects the deviation of the attention disengagement probability for fear from the mean level of attention disengagement for other facial emotional stimuli at a given age.

Importantly, comparing the magnitude of this score across the age groups can be used to estimate whether the amount of relative attentional bias to fearful faces increases with age.

As the HR data were normally distributed, the HR change scores were subjected to a $3 \times 5 \times 4$ repeated measures analysis of variance (ANOVA) with Emotion (neutral, happy, fearful) and Time (0-500, 500-1000, 1000-1500, 1500-2000, 2000-2500 ms) as 
within-subject factors and Age $(5,7,9,11)$ as a between-subjects factor. In all analyses, Bonferroni correction was applied for the $p$-values in cases where the number of pairwise comparisons was higher than three.

\section{Results}

Behavioral data. Across facial expressions, age differences were observed in the overall probability in disengaging attention from faces, $\chi 2=7.80, p<.05, \eta^{2}=.07$ (Kruskal-Wallis test). Mann-Whitney U-tests showed that disengagement probability decreased markedly after 5 months of age, with the 5-month-olds $(M=.74)$ differing from 7-month-olds $(\mathrm{M}=.62), \mathrm{Z}=-1.94, p \leq .05,9$-month-olds $(\mathrm{M}=.56), \mathrm{Z}=-2.42, p<$ .05 , and 11 -month-olds $(\mathrm{M}=.57), \mathrm{Z}=-2.28, p<.05$. Friedman tests revealed that the age groups differed in the effects of facial expressions on disengagement probability (Figure 2a). There were no main effects of facial expressions in the 5-month-olds, $F_{R}=3.91, d f=$ $2, p>.14, \mathrm{~W}=.08$, and the 11 -month-olds, $F_{R}=3.98, d f=2, p>.13, \mathrm{~W}=.08$, whereas significant effects of facial expressions on disengagement probability were observed in the 7-month-olds, $F_{R}=7.77, d f=2, p<.05, \mathrm{~W}=.15$, and the 9-month-olds, $F_{R}=14.22$, $d f=2, p<.01, \mathrm{~W}=.25$. Pairwise comparisons with Wilcoxon tests indicated that in the 7-month-olds, disengagement probability was significantly lower for fearful than happy faces, $z=-3.12, p<.01$, whereas the differences between fearful and neutral or happy and neutral expressions failed to reach significance, $p s>.08$. In the 9-month-olds, fearful faces resulted in a lower disengagement probability than happy, $z=-2.91, p<.01$, and neutral faces, $z=-3.15, p<.01$, with no differences between happy and neutral faces, $z=$ $-0.92, p>.35$. A direct comparison of the fear bias scores (see Figure 4a) between all age 
groups did not, however, result in a significant effect of age on the magnitude of the relative attentional bias to fearful faces, $\chi^{2}=4.43, p>.21, \eta^{2}=.04$.

\section{Insert Figure 2 about here}

HR data. The mean heart rate during the prestimulus baseline period did not differ between the facial expression conditions, $F(2,174)=1.06, p>.32$, nor was there an Emotion $\times$ Age interaction, $F(6,87)=.70, p>.65$. A main effect of Age, $F(3,87)=$ $8.95, p>.001, \eta_{\mathrm{p}}{ }^{2}=.24$, indicated that in general, HR decreased with age. A $3 \times 5 \times 4$ ANOVA on the HR change scores during the Overlap trials revealed a main effect of Time, $F(4,348)=27.69, p<.001, \eta_{\mathrm{p}}^{2}=.24$, and an Emotion $\times$ Time interaction, $F(8$, $696)=3.01, p<.05, \eta_{\mathrm{p}}^{2}=.03$, but no interactions involving Age, $p \mathrm{~s}>.11$. The Emotion $\times$ Time interaction reflected the fact that the HR deceleration became more pronounced during fearful than happy and neutral trials during the course of stimulus presentation (Figure 3). To reduce the number of paired comparisons, fearful faces were contrasted with non-fearful (i.e., an average of happy and neutral) faces to compare the strength of HR deceleration within each time window. Paired sample t-tests with Bonferroni correction revealed a difference between fearful and non-fearful faces only during the last time window $(2000-2500 \mathrm{~ms}), t(90)=-2.63, p<.05$, all other $p \mathrm{~s}>.5$. An inspection of the effects separately within each age group showed that the Emotion $\times$ Time interaction was significant in the 5-month-olds, $F(8,184)=3.29, p<.05, \eta_{\mathrm{p}}{ }^{2}=.13$. The Emotion $\times$ Time interactions were not significant in 7-, 9-, and 11-month-old infants, all $p \mathrm{~s}>.15$, 
although a similar trend toward more pronounced HR deceleration during fearful face trials was observed in all age groups.

\section{Insert Figure 3 about here}

\section{Discussion}

The data from Experiment 1 showed age-related differences in the behavioral measures of attention disengagement from fearful, happy, and neutral faces. The infants aged 7 and 9 months were less likely to disengage their attention from fearful faces, whereas the effects of facial expressions on disengagement probability were absent in infants aged 5 and 11 months. The HR data showed a more pronounced and longerlasting HR deceleration during fearful than happy and neutral trials. This effect did not, however, show an interaction with the infants' age and appeared to reflect a more generalized influence of fearful faces on HR deceleration across the age groups, although separate tests showed a significant effect only in the 5-month-old infants. The eye movement data provided support for the hypothesis that the attentional bias to fearful faces emerges between 5 and 7 months of age. The direct comparison of the relative fear bias scores (i.e., the probability of disengaging attention from fearful faces subtracted from the average disengagement probability score for happy and neutral faces) between the age groups was not significant, however. To provide a further test of the emergence of the attentional bias to fearful faces between 5 and 7 months of age, we analyzed additional data from an independent sample of infants tested longitudinally in Experiment 2. 


\section{Experiment 2}

\section{Methods}

Participants. The participants in the present analyses were a subsample of infants in an ongoing longitudinal cohort study, started in April 2012. All infants tested at 5 and 7 months of age by September $5^{\text {th }}, 2012$ were included in the present analyses. The final sample included in the analyses consisted of 41 healthy and full-term ( $\geq 37$ weeks) infants (18 girls) who were tested at both 5 (mean age $=152$ days, $S D=3.70)$ and 7 (mean age $=$ 213 days, $S D=3.25$ ) months of age. Nine additional infants participated in the study but were excluded from all analyses because of pre-term birth $(n=1)$, technical errors $(n=$ 1), not being tested at 7 months of age ( $n=1$, due to moving) and for providing $<2$ scorable trials in one or more stimulus condition $(n=6)$. All of the participants came from urban, middle class families of Finnish origin. Approval for the project was obtained from the Ethical Committee of Tampere University Hospital and an informed, written consent was obtained from the parent of each child.

Stimuli and procedure. As a part of the longitudinal study, infants' attention to facial expressions were examined at 5 and 7 months of age by using the Overlap paradigm (see Leppänen et al., 2011 for details of the methodology). In a difference to Experiment 1 and previous studies (Leppänen et al., 2010, 2011), the present experiment was started by net placement for the recording of EEG (Electrical Geodesics, Inc., Eugene, OR, USA) and a calibration procedure for corneal-reflection eye-tracking (Tobii TX300, Tobii Technology, Sweden). Following successful net placement and calibration (or three unsuccessful attempts to calibrate the eye-tracker), the infants were presented with the Overlap paradigm. The procedure and stimuli in the Overlap paradigm were 
similar to those used in Experiment 1, with the following exceptions: a) the stimulus onset asynchrony between the face and the peripheral stimulus was $1000 \mathrm{~ms}$ and the peripheral stimulus was presented for $3000 \mathrm{~ms}$, b) monkey faces were not shown but a phase-scrambled face was presented as a non-face control stimulus, and c) a total of 24 trials (6/condition) were run in the first part of the testing session. The present analyses are based on the disengagement probabilities from neutral, happy, and fearful facial expressions during the first part of the experiment (the second part of stimulus presentation was added for the purposes of EEG measurement and will be reported separately).

Analysis of the behavioral data. Visual attention was analyzed from video recordings of infants' eye movements during the presentation of stimuli and the procedures for calculating disengagement probabilities from fearful, happy, and neutral faces were identical to Experiment 1 . The mean number of scorable trials included in the analyses was 16.28 (fearful $=5.45$; happy $=5.38$; neutral $=5.45)$, with no significant difference in the number of scorable trials between the 5- and 7-month measurements, $t(40)=0.85, p>.40$. The statistical analyses of the eye movement data were conducted in an identical fashion to Experiment 1.

\section{Results}

The overall probability of attention disengagement (i.e., an average of fearful, happy, and neutral trials) did not differ between the 5- and 7-month measurements $(M=$ .79 and .77 , respectively), $z=-1.09, p>.27$. Again, significant effects of facial expressions on disengagement probability were observed at 7 months of age, $F_{R}=18.96$, $d f=2, p<.001, \mathrm{~W}=.23$, but not at 5 months of age, $F_{R}=3.98, d f=2, p>.13, \mathrm{~W}=.05$. 
(see Figure 2b). The significant effect at 7 months of age was due to disengagement probability being lower for fearful than happy, $z=-3.60, p<.001$, and neutral faces, $z=-$ $3.95, p<.001$, whereas no difference between happy and neutral faces was found, $z=-$ $0.70, p>.48$. A comparison of the relative fear bias scores between the two measurement points (Figure 4b) also indicated that the magnitude of the attentional bias to fearful faces increased significantly from 5 to 7 months of age, $z=-2.02, p<.05$ (Wilcoxon test).

\section{Insert Figure 4 about here}

\section{Discussion}

Experiment 2, using a within-subjects design, provided strong evidence for a developmental change in attention to fearful facial expressions between 5 and 7 months of age. Whereas at 5 months of age, facial expression of the central stimulus did not have an effect on infants' probability of disengaging attention, the same infants at 7 months of age were significantly more likely to maintain their attention on fearful as compared to happy and neutral faces throughout the trial. With different samples of infants and experiments employing both between- and within-subjects designs, Experiments 1 and 2 converge in providing no evidence for an overt attentional bias to fearful faces in 5month-old infants, whereas the evidence for an attentional bias in 7-month-old infants is clear.

\section{General Discussion}

The present research investigated the potential developmental changes in patterns of attention to fearful, happy, and neutral faces between 5 and 11 months of age. Based 
on previous research, we hypothesized that the attentional bias to fearful faces would appear between 5 and 7 months of age and remain evident also at 9 and 11 months of age. The attentional bias was expected to manifest in a decreased probability of disengaging attention from fearful faces and in a larger attention-related HR deceleration to fearful faces from 7 months onwards.

The results supported our hypothesis regarding the emergence, but not the stability of the attentional bias. First, the emergence of an attentional bias to fearful faces by 7 months of age was confirmed: facial expression did not have a significant effect on the probability of disengaging attention in the two samples of 5-month-old infants, whereas 7- and 9-month-old infants had a lower probability of disengaging attention from fearful than non-fearful faces. According to Experiment 1, the attentional bias appeared to have dissipated by 11 months of age. Across the age groups, the HR data (Experiment 1) showed a more pronounced and longer-lasting HR deceleration during fearful than happy and neutral trials, although this effect was significant only in the 5-month-olds' group. In Experiment 1, the probability of disengaging attention from facial stimuli toward peripheral stimuli decreased from 5 to 7 months of age, after which the probability remained constant. This difference between 5 and 7 months of age was not, however, replicated in Experiment 2.

The observed emergence of the attentional bias to fearful faces between 5 and 7 months of age is in line with previous research (Peltola, Leppänen, Mäki et al., 2009) and with the model of the development of emotion-processing put forward by Leppänen and Nelson $(2009,2012)$. At the turn of the second half of the first year, major transitions in emotional development are observed. As infants progress towards the ability to move 
independently (typically between 6 and 8 months of age; Adolph, Vereijken, \& Denny, 1998), increasing their distance and time away from the caregivers, it becomes increasingly important to be able to detect and direct attention to potential signals of threat and danger emanating from others' facial, vocal, or bodily reactions. In parallel with changes in infants' motor abilities, caregivers typically start to show increased variability in their expressive behaviors toward the infant, including a heightened propensity to display emotionally negative facial signals in attempting to regulate the infant's actions in situations involving potential harm (Campos, Kermoian, \& Zumbahlen, 1992). As a result, facial expressions begin to gain more direct referential significance, which may enable infants to form associations between emotional signals and different contextual events. Whether or not the development of independent locomotion had an influence on the present pattern of results is not known, however, as data about the participants' motor abilities were not collected. To date, there are no studies that would have tracked the development of emotional face-processing abilities in parallel with motor development.

It is intriguing to note that there appears to be a developmental correspondence between the increase in infants' attentiveness to fearful facial signals and the onset of emotional fear responses such as fear of heights (Campos et al., 1992) and wariness toward strangers (Braungart-Rieker, Hill-Soderlund, \& Karrass, 2010). Cross-species data are available to suggest that it is a common pattern for the offspring of altricial species to show an apparent inability to respond fearfully to conspecifics or form threatrelated associations toward the caregiver in the early phases in development (Moriceau \& Sullivan, 2005). Only after reaching a certain developmental phase that is linked with an 
increase in independent locomotion and functional maturation of the amygdala do the species-typical fear responses begin to emerge. Such developmental transitions in fearrelated behaviors have been documented in monkeys (Bauman \& Amaral, 2008) and in depth in rodents (Moriceau, Roth, \& Sullivan, 2010). Sullivan and colleagues (Moriceau \& Sullivan, 2005; Moriceau et al., 2010) have argued that the absence of fear-learning during early development may function to optimize and protect the infant's attachment formation and proximity-seeking to the caregiver and, conversely, to prevent learning aversion toward the caregiver. Whether or not a similar inhibition of fear-learning - that might also account for the absence of an overt attentional bias to fearful facial signals in the youngest age group in the present study - exists in human infants until the age of 5 to 7 months remains to be tested experimentally.

In Experiment 1, the HR data did not conform to our hypothesis in that there was no interaction between age and the strength of cardiac deceleration. Across age groups, there appeared to be a pronounced HR deceleration to fearful faces, although separate tests showed a significant effect only in the 5-month-old infants. While the reasons for the absence of significant effects in separate tests with the other age groups are not clear, the pattern of results nevertheless raises an interesting possibility of a dissociation between the attentional measures based on eye movements and heart rate. It might thus be that the influences of emotional expressions on overt attention disengagement require functional interaction between limbic circuits providing modulatory signals related to the emotional significance of stimuli (i.e., the amygdala) and areas controlling attentional focus (i.e., PFC), and that such functional connectivity may not be sufficiently matured until the age of 5 to 7 months (Leppänen \& Nelson, 2009, 2012). The HR deceleration 
response to threat-related stimuli, on the other hand, may more closely reflect an obligatory, subcortically mediated attentional orienting response that is less dependent on cortical processing (Bradley, 2009; Lang \& Bradley, 2010). The subcortical structures enabling infants to respond with cardiac deceleration to various stimuli (e.g., simple auditory stimuli and visual figures) appear to be functional already during the newborn period (Graham \& Jackson, 1970; Reynolds \& Richards, 2007; Sameroff, Cashmore, \& Dykes, 1973). Therefore, it may be feasible to suggest that also the neural circuitry responsible for producing the autonomic response to stimuli conveying potential emotional significance (i.e., connections from the amygdala to the brainstem structures controlling autonomic responses) may mature considerably earlier than the amygdalocortical circuitry enabling controlled attentiveness to emotional signals and producing the types of behavioral effects observed in the present experiments.

This interpretation of the HR data potentially reflecting immature connectivity between the PFC and limbic system parallels the model of attention development and emotion regulation by Ruff and Rothbart (1996) who make a distinction between the first and the second attention system. The first system, operating from birth, includes structures of the brainstem and the parietal cortex which act to produce changes in alertness (via the autonomic nervous system) and promote visual exploration by enabling spatial orienting to novel objects in the environment. The second attention system, operating through the frontal cortex, develops increasingly during the second half of the first year and many years after. The functions of the second attention system include exerting inhibitory control over reflexive response tendencies and integrating emotional significance into the guidance of attention. This type of endogenous attention control has 
been shown to develop rapidly during the second half of the first year (Colombo, 2001;

Kannass, Oakes, \& Shaddy, 2006; Ruff \& Rothbart, 1996), enabling infants to inhibit

unwanted attention shifts and to prioritize interesting stimuli. The developmental

progression from the first attention system to more endogenously controlled attention

may also be seen in the pattern of age-related changes in the overall attention

disengagement (i.e., across facial expressions) in Experiment 1. The peripheral stimuli

produced shifts of attention to their location on a majority of trials in 5-month-old infants, whereas infants from 7 to 11 months of age were more likely to maintain their attention on the central stimulus. The increase in self-regulated control of attention is attributable

to the maturation of prefrontal cortical structures which undergo major structural and functional maturational changes especially during the second half of the first year (Chugani \& Phelps, 1986; Matsuzawa et al., 2001). However, similar changes in overall attention disengagement were not replicated in Experiment 2, limiting the interpretability of the results from Experiment 1.

Akin to models relating the transition from reflexive to more controlled attention to the maturation of prefrontal cortical structures (e.g., Ruff \& Rothbart, 1996), Leppänen and Nelson $(2009,2012)$ suggested that the shift in attentiveness to fear-related signals during the second half-year of life is related to a functional maturation of a neural circuitry that is responsible for directing attention to emotionally significant signals (i.e., amygdala-PFC connectivity). The putative maturation of this system coincides with an increased distance and time away from the caregiver (due to motor development) and may even give rise to a sensitive period during which infants "expect" exposure to threatalerting cues and show heightened readiness to process and store information about 
associations between emotions and objects in the environment (Leppänen \& Nelson, 2012). In theory, such a sensitive period for processing fear-related information could account for the pronounced attentiveness to fearful faces at 7 and 9 - but not at $11-$ months of age. Another interpretation of the absence of a fear-related attentional bias in the 11-month-olds could be that the more advanced capacity for emotion regulation enables older infants to orient away from potential sources of distress such as fearful faces, possibly owing to the strengthened connectivity of the PFC to the limbic system (cf. Ruff \& Rothbart, 1996). It is important to note, however, the results by Nakagawa and Sukigara (2012) who found a longitudinally stable effect of lower disengagement probabilities to fearful than happy and neutral faces from 12 to 36 months of age. Their results strongly suggest that the attentional bias to fearful expressions, emerging between 5 and 7 months of age, is an enduring phenomenon and, therefore, the absence of significant effects in the 11-month-old infants in the present study should be interpreted cautiously.

In conclusion, the present study adds to the growing body of data indicating a transition in processing emotional information at around 5 to 7 months of age (Hoehl \& Striano, 2010; Leppänen \& Nelson, 2009, 2012; Peltola, Leppänen, Mäki et al., 2009).

Attention was allocated to fearful faces preferentially in 7- and 9-month-old, but not in 5month-old infants. The emerging prioritization of emotional saliency in processing social signals may provide a crucial scaffold for an infant with a newly found level of independence to navigate in an increasingly complex social and physical environment. A replication is needed to ascertain whether the pattern of results in the 11-month-old infants represents a genuine effect (i.e., an absence of attentional bias to fearful faces) or 
whether the attentional bias remains present over time (cf. Nakagawa \& Sukigara, 2012). A promising agenda for future studies is to determine the importance of individual differences in attentional biases and developmental timing, i.e., whether variations in the magnitude and the age of onset of the attentional bias to threat-related signals are relevant in relation to later social and emotional outcomes. 


\section{References}

Adolph, K. E., Vereijken, B., \& Denny, M. A. (1998). Learning to crawl. Child Development, 69, 1299-1312.

Aslin, R. N., \& Salapatek, P. (1975). Saccadic localization of visual targets by the very young human infant. Perception and Psychophysics, 17, 293-302.

Bauman, M. D., \& Amaral, D. G. (2008). Neurodevelopment of social cognition. In C. A. Nelson, \& M. Luciana (Eds.), Handbook of developmental cognitive neuroscience (2nd ed., pp. 161-185). Cambridge, MA: MIT Press.

Blaga, O. M., \& Colombo, J. (2006). Visual processing and infant ocular latencies in the overlap paradigm. Developmental Psychology, 42, 1069-1076.

Bornstein, M. H., \& Arterberry, M. E. (2003). Recognition, discrimination and categorization of smiling by 5-month-old infants. Developmental Science, 6, 585599.

Bradley, M. M. (2009). Natural selective attention: Orienting and emotion. Psychophysiology, 46, 1-11.

Bradley, M. M., Lang, P. J., \& Cuthbert, B. N. (1993). Emotion, novelty, and the startle reflex: Habituation in humans. Behavioral Neuroscience, 107, 970-980. 
Braungart-Rieker, J. M., Hill-Soderlund, A. L., \& Karrass, J. (2010). Fear and anger reactivity trajectories from 4 to 16 months: The roles of temperament, regulation, and maternal sensitivity. Developmental Psychology, 46, 791-804.

Campos, J. J., Kermoian, R., \& Zumbahlen, M. R. (1992). Socioemotional transformations in the family system following infant crawling onset. In N. Eisenberg, \& R. A. Fabes (Eds.), Emotion and its regulation in early development (pp. 25-40). San Francisco, CA: Jossey-Bass.

Chugani, H., \& Phelps, M. (1986). Maturational changes in cerebral function in infants determined by 18FDG positron emission tomography. Science, 231, 840-843.

Colombo, J. (2001). The development of visual attention in infancy. Annual Review of Psychology, 52, 337-367.

de Haan, M., Belsky, J., Reid, V., Volein, A., \& Johnson, M. H. (2004). Maternal personality and infants' neural and visual responsivity to facial expressions of emotion. Journal of Child Psychology and Psychiatry, 45, 1209-1218.

Farroni, T., Menon, E., Rigato, S., \& Johnson, M. H. (2007). The perception of facial expressions in newborns. European Journal of Developmental Psychology, 4, 2-13.

Graham, F. K., \& Clifton, R. K. (1966). Heart-rate change as a component of the orienting response. Psychological Bulletin, 65, 305-320.

Graham, F. K., \& Jackson, J. C. (1970). Arousal systems and infant heart rate responses. Advances in Child Development and Behavior, 5, 59-117. 
Grossmann, T., Johnson, M. H., Vaish, A., Hughes, D., Quinque, D., Stoneking, M., et al. (2011). Genetic and neural dissociation of individual responses to emotional expressions in human infants. Developmental Cognitive Neuroscience, 1, 57-66.

Hoehl, S., \& Striano, T. (2010). The development of emotional face and eye gaze processing. Developmental Science, 13, 813-825.

Hunnius, S., \& Geuze, R. H. (2004). Gaze shifting in infancy: A longitudinal study using dynamic faces and abstract stimuli. Infant Behavior and Development, 27, 397-416.

Kagan, J., \& Herschkowitz, N. (2005). A young mind in a growing brain. Mahwah, NJ: Lawrence Erlbaum Associates.

Kannass, K. N., Oakes, L. M., \& Shaddy, D. J. (2006). A longitudinal investigation of the development of attention and distractibility. Journal of Cognition and Development, 7, 381-409.

Kolassa, I. T., \& Miltner, W. H. (2006). Psychophysiological correlates of face processing in social phobia. Brain Research, 1118, 130-141.

Kotsoni, E., de Haan, M., \& Johnson, M. H. (2001). Categorical perception of facial expressions by 7-month-old infants. Perception, 30, 1115-1125.

LaBarbera, J. D., Izard, C. E., Vietze, P., \& Parisi, S. A. (1976). Four- and six-month-old infants' visual responses to joy, anger, and neutral expressions. Child Development, $47,535-538$. 
Lang, P. J., \& Bradley, M. M. (2010). Emotion and the motivational brain. Biological Psychology, 84, 437-450.

Leppänen, J. M., Moulson, M. C., Vogel-Farley, V. K., \& Nelson, C. A. (2007). An ERP study of emotional face processing in the adult and infant brain. Child Development, $78,232-245$.

Leppänen, J. M., \& Nelson, C. A. (2009). Tuning the developing brain to social signals of emotions. Nature Reviews Neuroscience, 10, 37-47.

Leppänen, J. M., \& Nelson, C. A. (2012). Early development of fear processing. Current Directions in Psychological Science, 21, 200-204.

Leppänen, J. M., Peltola, M. J., Mäntymaa, M., Koivuluoma, M., Salminen, A., \& Puura, K. (2010). Cardiac and behavioral evidence for emotional influences on attention in 7-month-old infants. International Journal of Behavioral Development, 34, 547-553.

Leppänen, J. M., Peltola, M. J., Puura, K., Mäntymaa, M., Mononen, N., \& Lehtimäki, T. (2011). Serotonin and early cognitive development: Variation in the tryptophan hydroxylase 2 gene is associated with visual attention in 7-month-old infants. Journal of Child Psychology and Psychiatry, 52, 1144-1152.

LoBue, V., \& DeLoache, J. S. (2010). Superior detection of threat-relevant stimuli in infancy. Developmental Science, 13, 221-228.

Malatesta, C. Z., \& Haviland, J. M. (1982). Learning display rules: The socialization of emotion expression in infancy. Child Development, 53, 991-1003. 
Matsuzawa, J., Matsui, M., Konishi, T., Noguchi, K., Gur, R. C., Bilker, W., et al. (2001). Age-related volumetric changes of brain gray and white matter in healthy infants and children. Cerebral Cortex, 11, 335-342.

Moriceau, S., Roth, T. L., \& Sullivan, R. M. (2010). Rodent model of infant attachment learning and stress. Developmental Psychobiology, 52, 651-660.

Moriceau, S., \& Sullivan, R. M. (2005). Neurobiology of infant attachment. Developmental Psychobiology, 47, 230-242.

Nakagawa, A., \& Sukigara, M. (2012). Difficulty in disengaging from threat and temperamental negative affectivity in early life: A longitudinal study of infants aged 12 to 36 months. Behavioral and Brain Functions, 8, 40.

Nelson, C. A., \& de Haan, M. (1996). Neural correlates of infants' visual responsiveness to facial expressions of emotion. Developmental Psychobiology, 29, 577-595.

Nelson, C. A., \& Dolgin, K. G. (1985). The generalized discrimination of facial expressions by seven-month-old infants. Child Development, 56, 58-61.

Peltola, M. J., Leppänen, J. M., \& Hietanen, J. K. (2011). Enhanced cardiac and attentional responding to fearful faces in 7-month-old infants. Psychophysiology, 48, 1291-1298.

Peltola, M. J., Leppänen, J. M., Mäki, S., \& Hietanen, J. K. (2009). Emergence of enhanced attention to fearful faces between 5 and 7 months of age. Social Cognitive and Affective Neuroscience, 4, 134-142. 
Peltola, M. J., Leppänen, J. M., Palokangas, T., \& Hietanen, J. K. (2008). Fearful faces modulate looking duration and attention disengagement in 7-month-old infants. Developmental Science, 11, 60-68.

Peltola, M. J., Leppänen, J. M., Vogel-Farley, V. K., Hietanen, J. K., \& Nelson, C. A. (2009). Fearful faces but not fearful eyes alone delay attention disengagement in 7month-old infants. Emotion, 9, 560-565.

Reynolds, G. D., \& Richards, J. E. (2007). Infant heart rate: A developmental psychophysiological perspective. In L. A. Schmidt, \& S. J. Segalowitz (Eds.), Developmental psychophysiology: Theory, systems, and methods (pp. 173-212). Cambridge, UK: Cambridge University Press.

Richards, J. E., \& Turner, E. D. (2001). Extended visual fixation and distractibility in children from six to twenty-four months of age. Child Development, 72, 963-972.

Ruff, H. A., \& Rothbart, M. K. (1996). Attention in early development: Themes and variations. New York, NY: Oxford University Press.

Sameroff, A. J., Cashmore, T. J., \& Dykes, A. C. (1973). Heart rate deceleration during visual fixation in human newborns. Developmental Psychology, 8, 117-119.

Todd, R. M., Evans, J. W., Morris, D., Lewis, M. D., \& Taylor, M. J. (2011). The changing face of emotion: Age-related patterns of amygdala activation to salient faces. Social Cognitive and Affective Neuroscience, 6, 12-23. 
Vaish, A., Grossmann, T., \& Woodward, A. (2008). Not all emotions are created equal: The negativity bias in social-emotional development. Psychological Bulletin, 134, 383-403.

Wilcox, B. M., \& Clayton, F. L. (1968). Infant visual fixation on motion pictures of the human face. Journal of Experimental Child Psychology, 6, 22-32. 


\title{
Footnotes
}

\begin{abstract}
${ }^{1}$ In the 5-month-olds' group, 3 infants were excluded from all data analyses due to technical errors resulting in unusable video files and 1 infant from the HR analyses due to a technical error resulting in missing HR data. In the 7-month-olds, 2 infants were excluded from all data analyses due to sleepiness $(n=1)$ and due to providing $<2$ trials in one or more stimulus condition $(n=1)$, and 7 infants from the HR analyses due to a technical error resulting in missing HR data. In the 9-month-olds, 1 infant was excluded from all data analyses due to a technical error resulting in an unusable video file and 1 infant from the HR analyses due to excessive artefacts in the ECG signal. In the 11month-olds, 1 infant was excluded from all data analyses due to providing $<2$ trials in one or more stimulus condition, and another 4 infants were excluded from the HR analyses due to excessive artefacts in the ECG signal.
\end{abstract}




\section{Figure Captions}

Figure 1. The sequence of events during a single trial of the Overlap paradigm in Experiment 1.

Figure 2. Disengagement probabilities from fearful, happy, and neutral expressions in different age groups in A) Experiment 1 and B) Experiment 2. $(* * p<.01 ; * * * p<.001)$.

Figure 3. The mean HR change during fearful, happy, and neutral face trials (averaged across the age groups) in Experiment 1.

Figure 4. The average relative fear bias scores (i.e., the average disengagement probability score for happy and neutral faces - the disengagement probability score for fearful faces) in different age groups in A) Experiment 1 and B) Experiment 2. (* $p<$ $.05)$. 


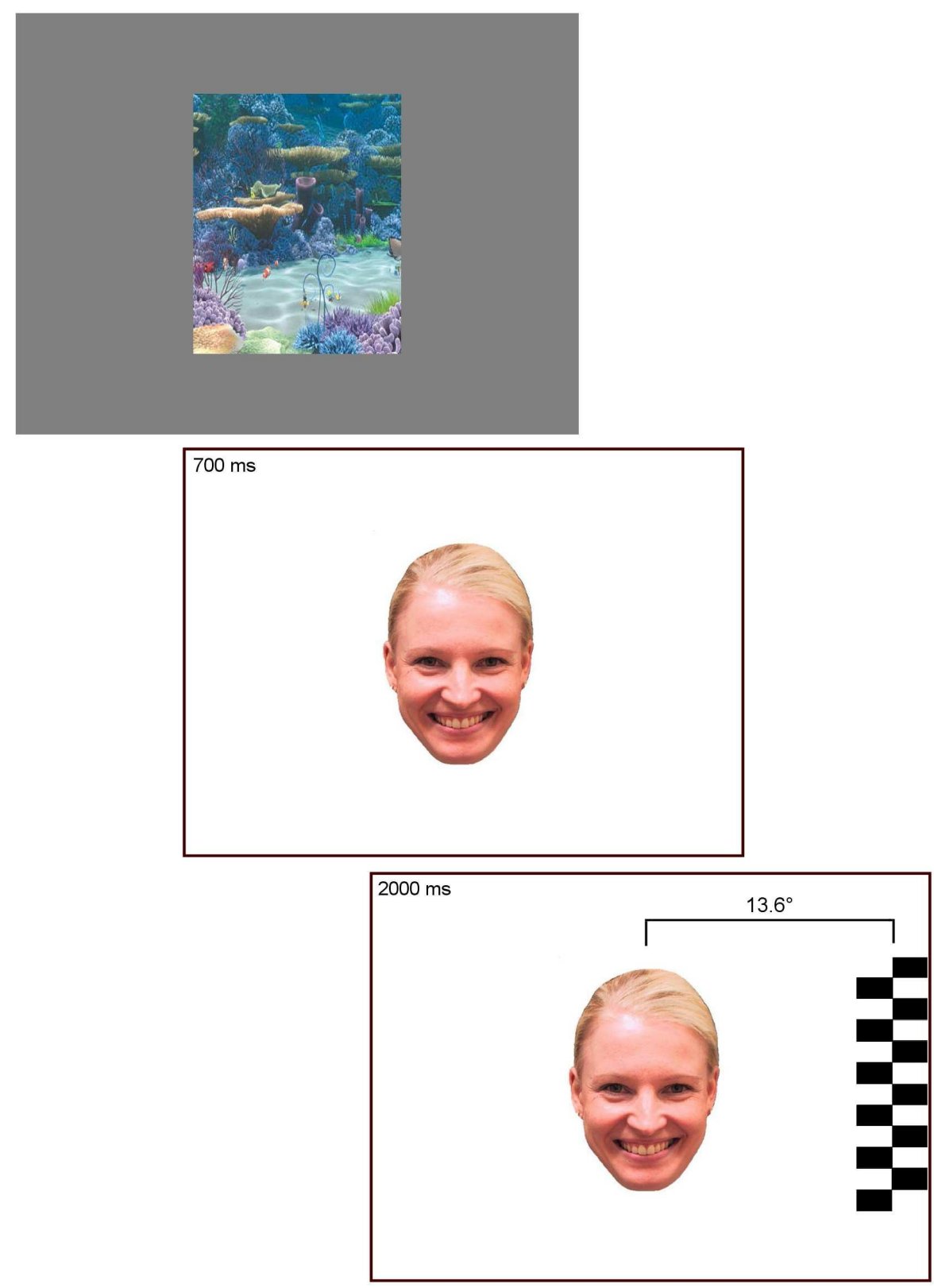

Figure 1. 

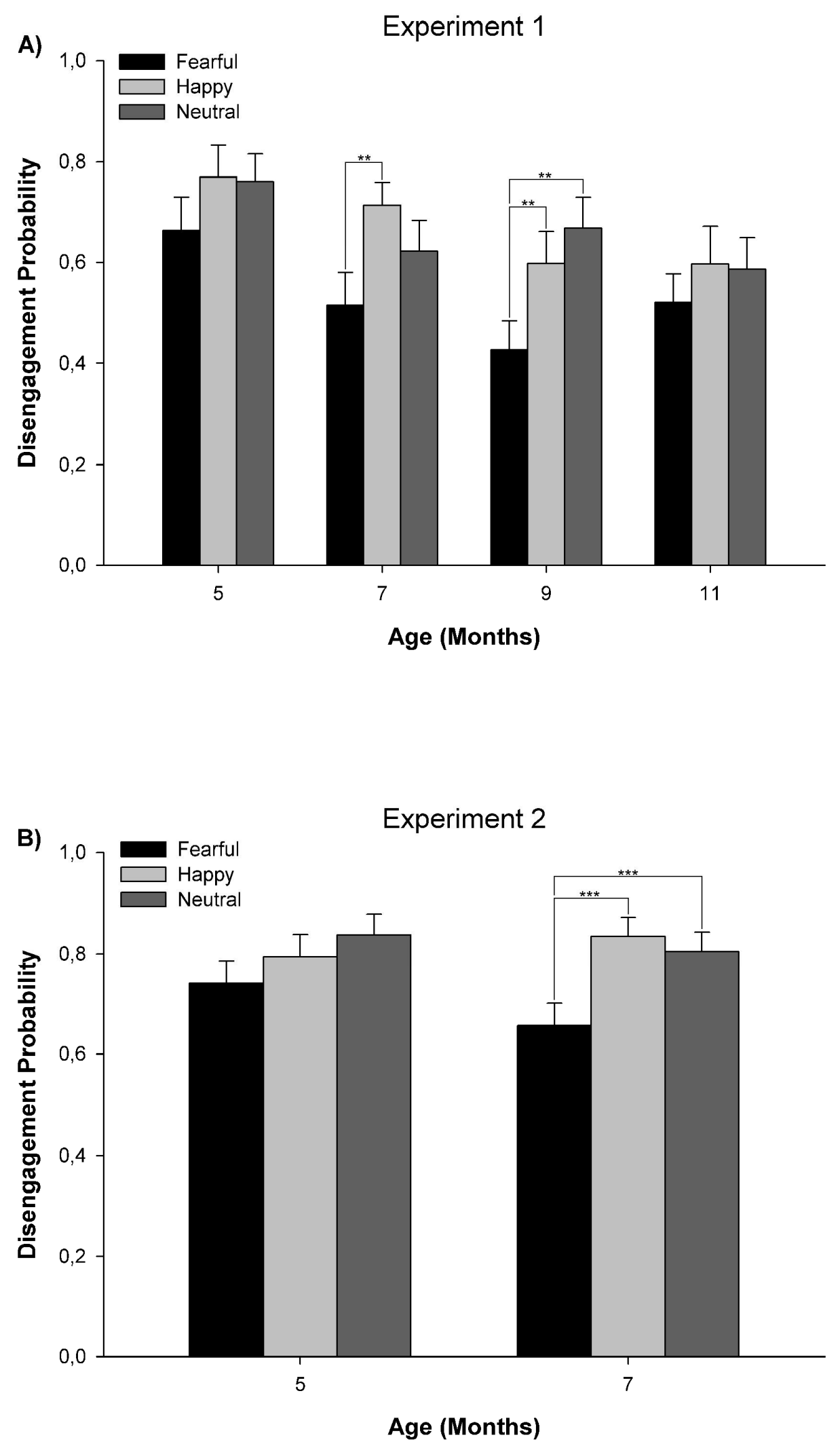

Figure 2. 
1

2

3

4

5

6

7

8

9

10

11

12

13

14

15

16

17

18

19

20

21

22

23

24

25

26

27

28

29

30

31

32

33

34

35

36

37

38

39

40

41

42

43

44

45

46

47

48

49

50

51

52

53

54

55

56

57

58

59

60

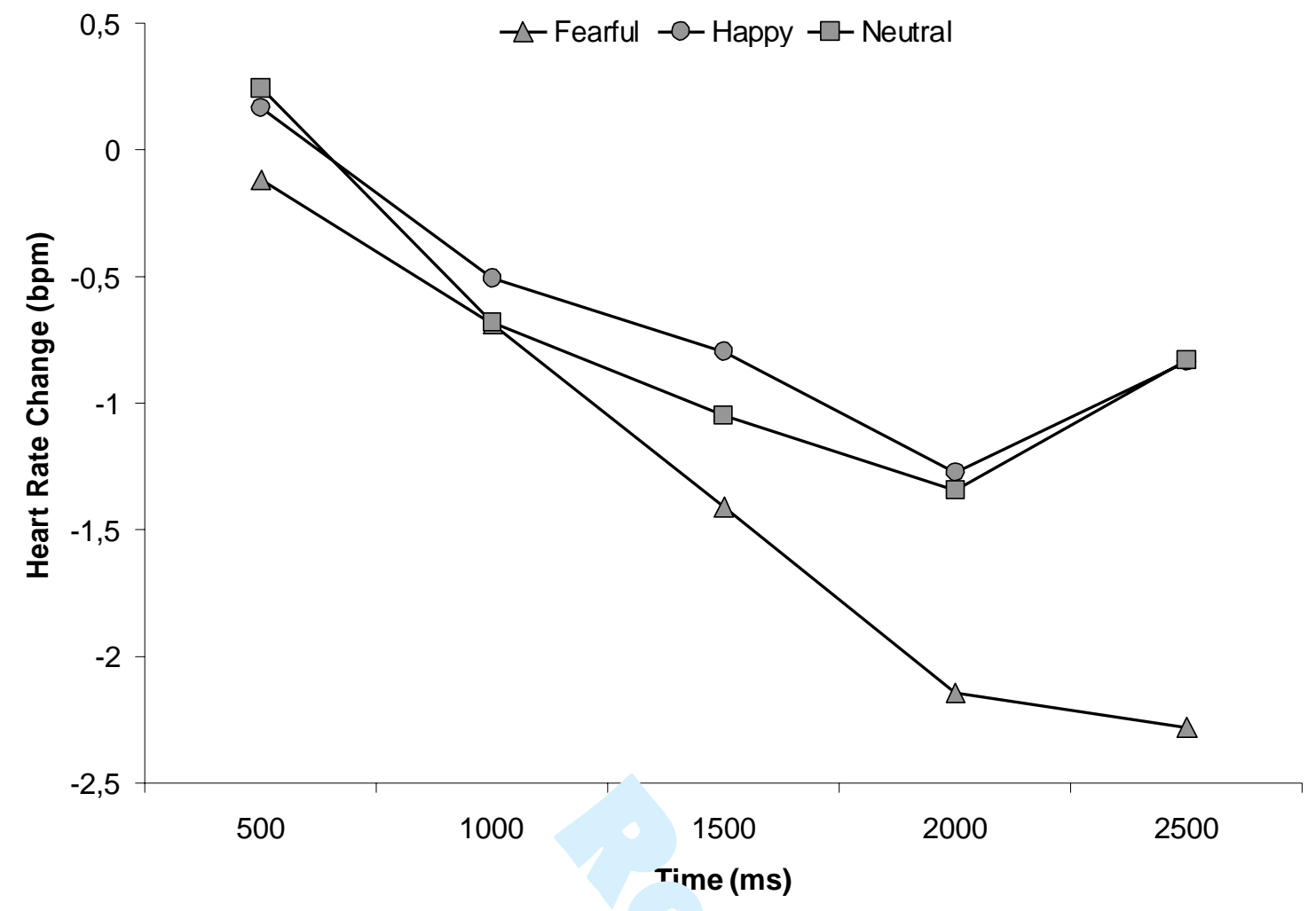

Figure 3. 


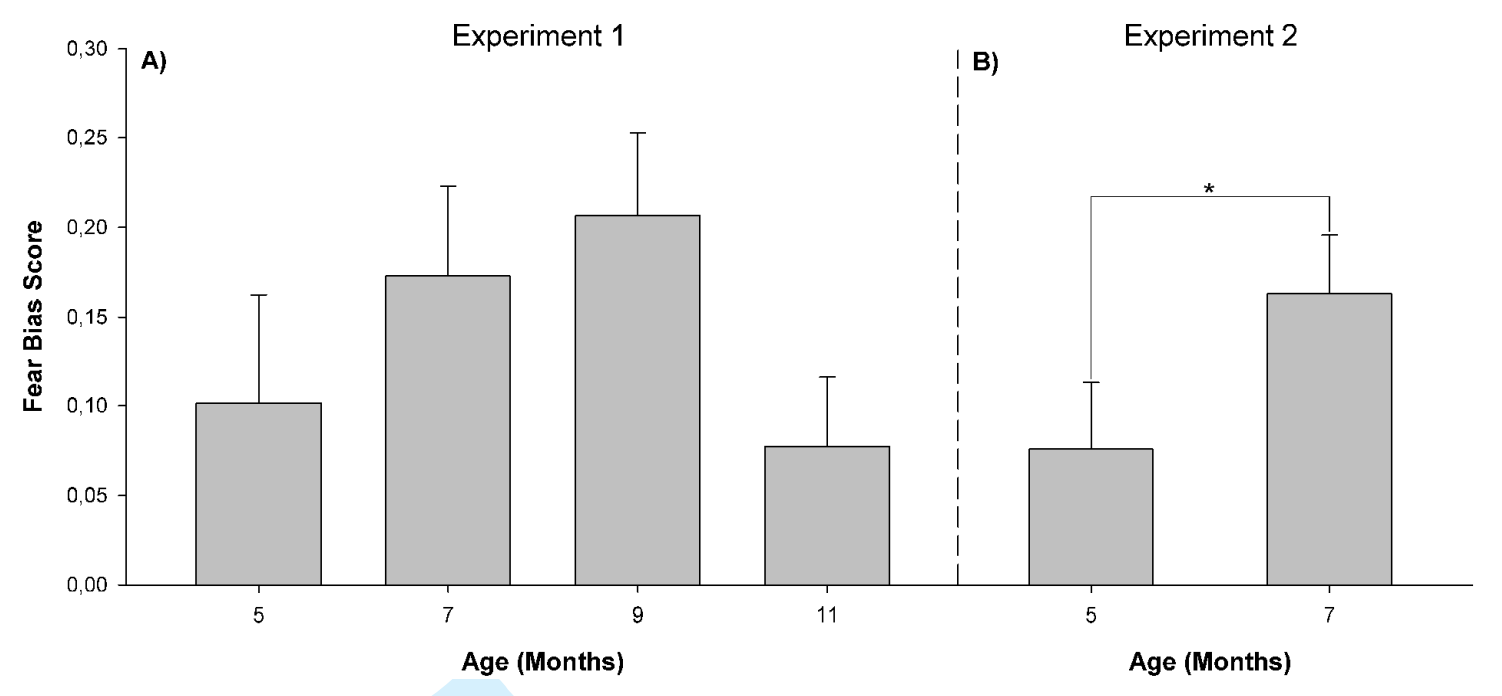

Figure 4. 

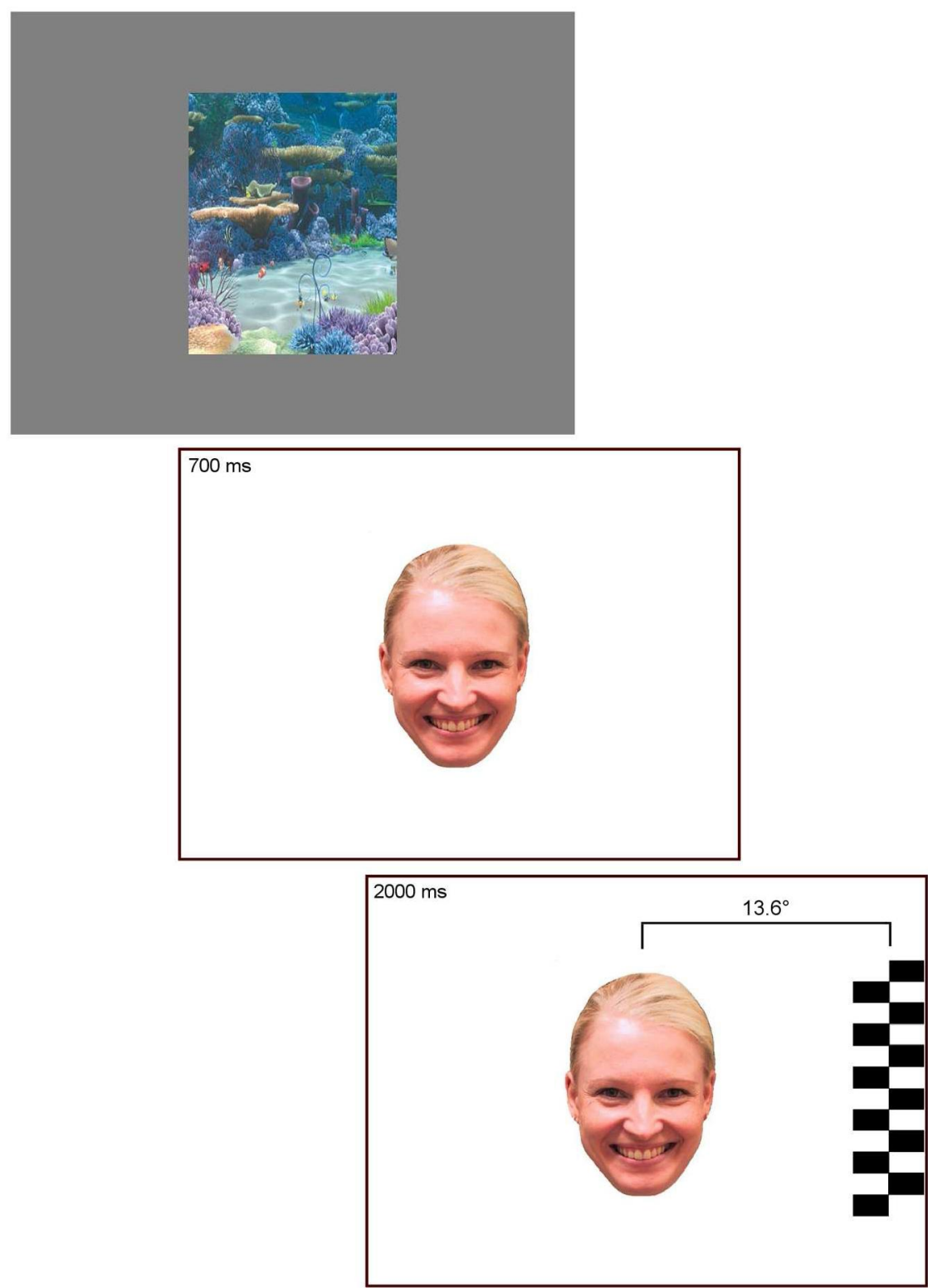

The sequence of events during a single trial of the Overlap paradigm in Experiment 1. $608 \times 841 \mathrm{~mm}(71 \times 71 \mathrm{DPI})$ 

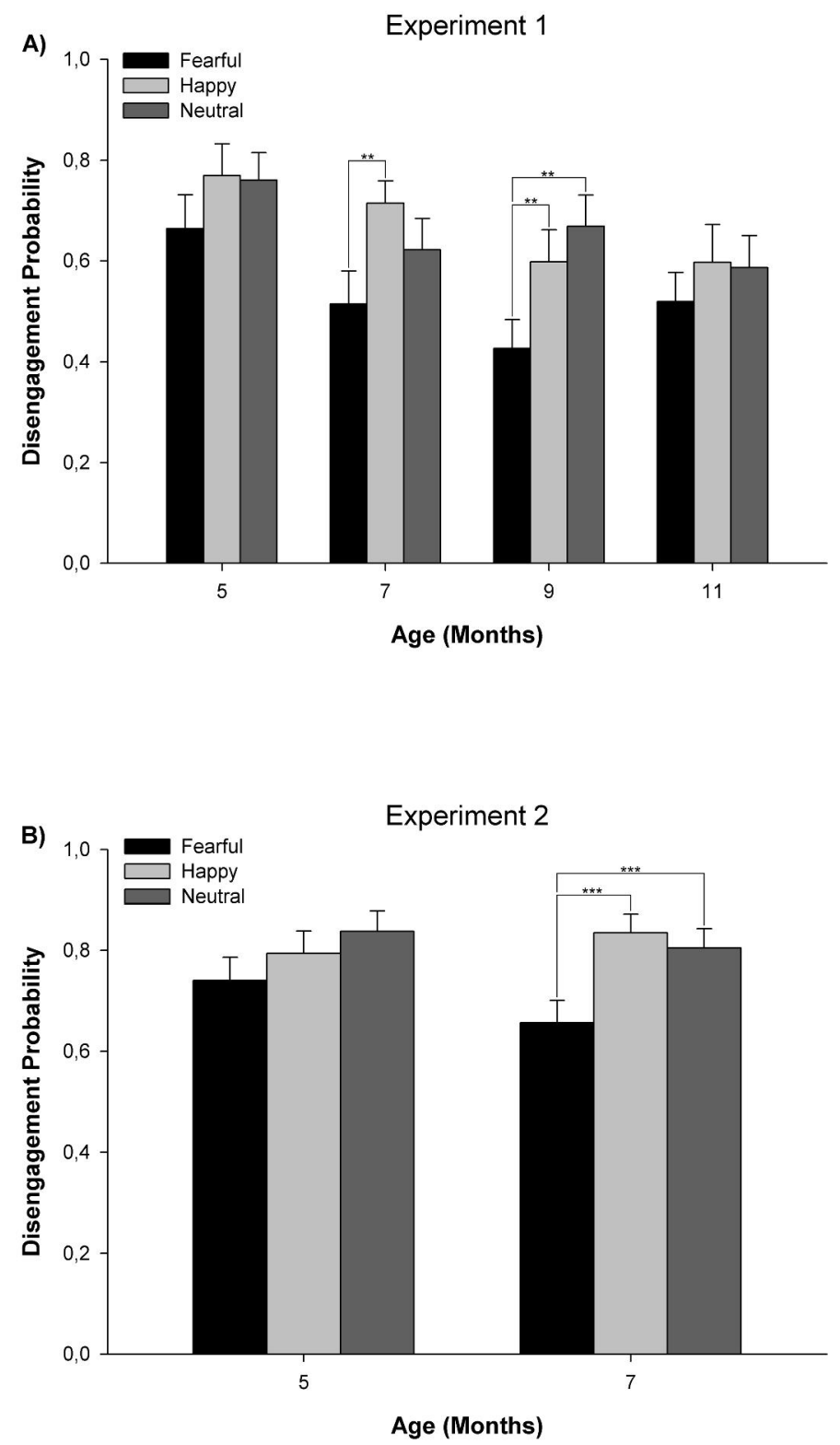

Disengagement probabilities from fearful, happy, and neutral expressions in different age groups in A) Experiment 1 and B) Experiment $2 .(* * \mathrm{p}<.01 ; * * * \mathrm{p}<.001)$. $254 \times 426 \mathrm{~mm}(300 \times 300 \mathrm{DPI})$ 
$0.5]$

Q

O

峁

ณ

닌

这

$\triangle$

1

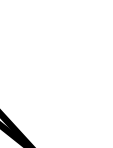

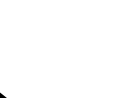

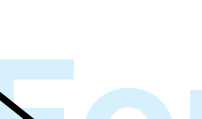<smiles>[I-]</smiles>

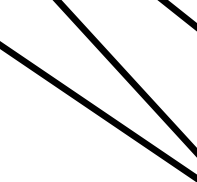
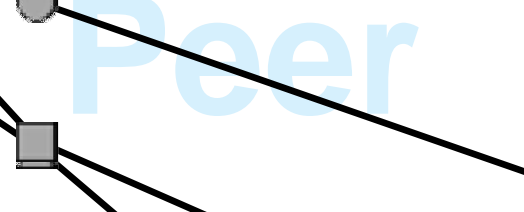

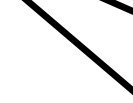

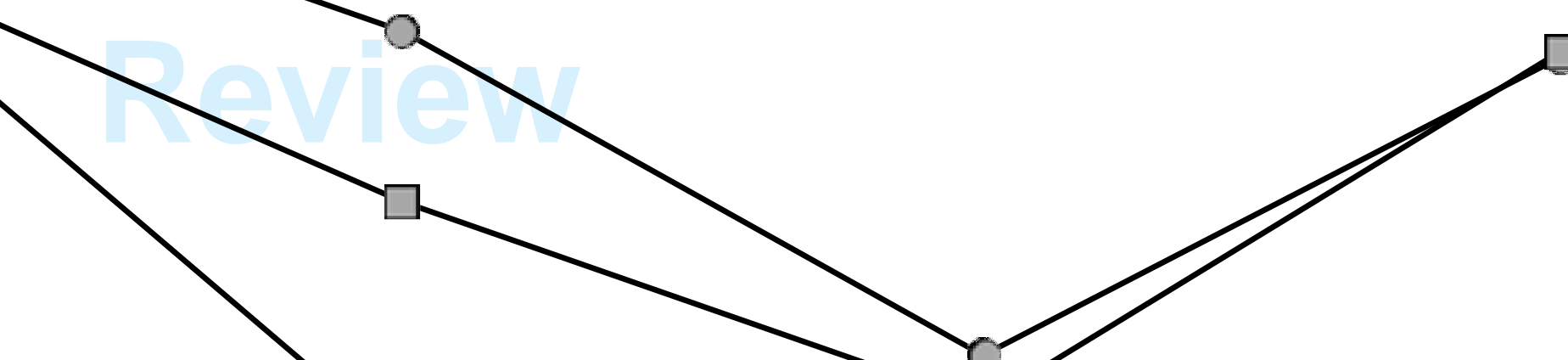

$-2.5$ 
The average relative fear bias scores (i.e., the average disengagement probability score for happy and neutral faces - the disengagement probability score for fearful faces) in different age groups in A) Experiment 1 and B) Experiment 2. (* $\mathrm{p}<.05)$.

$1549 \times 694 \mathrm{~mm}(96 \times 96 \mathrm{DPI})$ 